\title{
Status and prospects of searches for neutrinoless double beta decay
}

\author{
Bernhard Schwingenheuer \\ Max-Planck-Institut für Kernphysik, 69117 Heidelberg, Germany
}

\begin{abstract}
The simultaneous beta decay of two neutrons in a nucleus without the emission of neutrinos (called neutrinoless double beta decay) is a lepton number violating process which is not allowed in the Standard Model of particle physics. More than a dozen experiments using different candidate isotopes and a variety of detection techniques are searching for this decay. Some (EXO-200, Kamland-Zen, GERDA) started to take data recently. EXO and KamlandZen have reported first limits of the half life $T_{1 / 2}^{0 \nu}$ for ${ }^{136}$ Xe. After a decade of little progress in this field, many new results will soon scrutinize the claim from part of the HeidelbergMoscow collaboration to have observed this decay. The sensitivities of the different proposals are reviewed.
\end{abstract}

\section{Introduction}

For 35 isotopes, $\beta$ decay is energetically forbidden but double beta decay $(2 \nu \beta \beta)$ is allowed

$$
(A, Z) \rightarrow(A, Z+2)+2 e^{-}+2 \bar{\nu}_{e} .
$$

This process has been observed directly for 11 isotopes with half lives between $7 \cdot 10^{18} \mathrm{yr}$ and $2 \cdot 10^{21}$ yr [1, 2, 3].

Since neutrinos have no electric charge, there is no known symmetry which forbids that they mix with their anti-particles. As a consequence double beta decay without neutrino emission $(0 \nu \beta \beta)$ might occur as well which is predicted by almost all extensions of the Standard Model of particle physics (see e.g. [4, 5]). Its observation would imply that lepton number is violated since only electrons are emitted.

The experimental signature of $0 \nu \beta \beta$ is a line at the $Q_{\beta \beta}$ value of the decay if the sum of the electron energies is histogrammed.

Part of the Heidelberg-Moscow collaboration claims to have evidence for this line for ${ }^{76} \mathrm{Ge}$ with $T_{1 / 2}^{0 \nu}=\left(1.19_{-0.23}^{+0.37}\right) \cdot 10^{25} \mathrm{yr}[6]$ at a $4 \sigma$ level. In a more sophisticated analysis a significance beyond $6 \sigma$ was calculated [7. The current experiments using ${ }^{136} \mathrm{Xe}$ (EXO-200 and KamlandZen) and ${ }^{76} \mathrm{Ge}$ (GERDA) will scrutinize this result within the next 12 months.

In total more than a dozen large scale experimental programs are suggested or under construction to search for $0 \nu \beta \beta$. These programs are compared in this article and also the status of theoretical matrix element calculations is discussed. The latter are needed to convert an experimental measurement or limit on $T_{1 / 2}^{0 \nu}$ to a particle physics parameter. For general reviews the reader is referred to the literature [8, 4, 9, 5].

There are also other related processes like double positron decay or double electron capture. While $0 \nu \beta \beta$ is already a suppressed process, the other decays are expected to be even rarer 
unless there is some resonance enhancement [10, 11, 12, 5, 13]. In this article only $0 \nu \beta \beta$ decay searches are discussed.

\section{Motivation for $0 \nu \beta \beta$}

Since neutrinos have mass but no electric charge, there is no known symmetry which forbids additional terms in the effective Lagrangian besides the standard Dirac mass term $m_{D}$ [8, 4, 5, 14]:

$$
\begin{aligned}
L_{\mathrm{Yuk}} & =m_{D} \overline{\nu_{L}} \nu_{R}+\frac{1}{2} m_{L} \overline{\nu_{L}}\left(\nu_{L}\right)^{c}+\frac{1}{2} m_{R} \overline{\left(\nu_{R}\right)^{c}} \nu_{R}+\text { h.c. } \\
& =\frac{1}{2}\left(\overline{\nu_{L}}, \overline{\left(\nu_{R}\right)^{c}}\right)\left(\begin{array}{cc}
m_{L} & m_{D} \\
m_{D} & m_{R}
\end{array}\right)\left(\begin{array}{c}
\left(\nu_{L}\right)^{c} \\
\nu_{R}
\end{array}\right)+\text { h.c. }
\end{aligned}
$$

The subscript $L$ stands for the left-handed chiral field $\nu_{L}=\frac{1}{2}\left(1-\gamma_{5}\right) \nu$ and $R$ for the righthanded projection $\frac{1}{2}\left(1+\gamma_{5}\right) \nu$. The superscript $C$ denotes charge conjugation, i.e. $\nu^{C}$ stands for an (incoming) anti-neutrino. The $m_{R}$ term describes therefore an incoming neutrino $\nu_{R}$ and an outgoing anti-neutrino $\overline{\left(\nu_{R}\right)^{C}}$, i.e. this term violates lepton number by 2 units. The eigen states of the mass matrix are of the form $\left(\nu+\nu^{c}\right)$. Consequently neutrinos are expected to be - in general - their own anti-particles, i.e. Majorana particles.

Neutrinos (or anti-neutrinos) are produced in charged weak current reactions and - depending on the charge of the associated lepton - only one chiral projection couples ("V-A" current of weak interactions). For example in $\beta$ decay $n \rightarrow p e^{-} \bar{\nu}_{e, R}$, a right-handed anti-neutrino couples which can be decomposed in the mass eigen states $\nu_{i}$ and helicity $(h=\vec{\sigma} \vec{p})$ eigen states:

$$
\bar{\nu}_{e, R}=\bar{\nu}_{e} \frac{1}{2}\left(1+\gamma_{5}\right)=\sum_{i=1}^{3} U_{e i}\left(\bar{\nu}_{i, h=+1}+\frac{m_{i}}{E} \bar{\nu}_{i, h=-1}\right)
$$

Here, $U$ is the PMNS mixing matrix [15], $m_{i}$ are the mass eigen values, and $E$ is the neutrino energy.

For Dirac particles, only detection reactions like $p \bar{\nu}_{e, R} \rightarrow n e^{+}$are possible. If, on the other hand, neutrinos are massive Majorana particles, then the helicity suppressed component $\bar{\nu}_{i, h=-1}$ of $\bar{\nu}_{e, R}$ can undergo the reaction $n \nu_{e, L} \rightarrow p e^{-}$. Here

$$
\nu_{e, L}=\frac{1}{2}\left(1-\gamma_{5}\right) \nu_{e}=\sum_{i=1}^{3} U_{e i}\left(\nu_{i, h=-1}+\frac{m_{i}}{E} \nu_{i, h=+1}\right)
$$

Taking both processes involving neutrons together we have $2 n \rightarrow 2 p+2 e^{-}$(or better $\left.(A, Z) \rightarrow(A, Z+2)+2 e^{-}\right)$mediated by a massive Majorana neutrino with an effective coupling strength which is called the Majorana mass:

$$
m_{\beta \beta}=\left|\sum_{i=1}^{3} U_{e i}^{2} \cdot m_{i}\right|
$$

The helicity suppression $\left(m_{i} / E\right)^{2}$, which is e.g. $10^{-14}$ for a neutrino mass of $0.1 \mathrm{eV}$ and a neutrino energy of $1 \mathrm{MeV}$, is compensated by the large number of nuclei per mole.

Schechter and Valle showed that the observation of $0 \nu \beta \beta$ ensures that neutrinos have a Majorana component [16]. Recently it was pointed out that the "guaranteed" Majorana mass through radiative corrections is however only in the range of $10^{-24} \mathrm{eV}$ [17], i.e. negligible compared to the mass scales of neutrino oscillations [15]. Consequently, if $0 \nu \beta \beta$ is observed, 
other mechanisms like the exchange of supersymmetric particles or heavy Majorana neutrinos might be the dominating process and the known neutrino could even be (effectively) a Dirac particle. While for the initially motivated light neutrino exchange the coupling strength is proportional to $m_{i}$ (Eq. 6), the one for processes with heavy fermion exchange is proportional to $1 / M_{F}$ with $M_{F}$ being the mass of the exchange particle (provided that the helicity of the two leptonic currents are the same and $\left.M_{F}^{2}>q^{2}\right){ }^{1}$ In such processes, lepton number violation or lepton flavor violation can be accessible with accelerator experiments as well [18. If lower limits on $M_{F}$ from e.g. LHC are higher than typically $10 \mathrm{TeV}$, then the contributions to $0 \nu \beta \beta$ become smaller than the ones expected from light neutrino exchange with a mass of $0.05 \mathrm{eV}$ [4. The argument can be turned around: $0 \nu \beta \beta$ will provide also information on $\mathrm{TeV}$ scale physics.

The exchange of light neutrinos is discussed predominantly. Its strength depends on $U$ which can be parameterized by 3 rotation angles and 1 phase $\left(\theta_{12}, \theta_{13}, \theta_{23}\right.$, and $\delta$, all measurable by neutrino oscillation experiments), and 2 additional phases $\left(\alpha_{21}\right.$ and $\left.\alpha_{31}\right)$. The latter are called Majorana phases and influence processes like $0 \nu \beta \beta$. Oscillation experiments measure difference of squared masses $\left(\Delta m_{21}^{2}=m_{2}^{2}-m_{1}^{2}=\left(7.58_{-0.26}^{+0.22}\right) \cdot 10^{-5} \mathrm{eV}^{2}\right.$ and $\left|\Delta m_{31}\right|^{2}=\left|m_{3}^{2}-m_{1}^{2}\right|=$ $\left.\left(2.35_{-0.09}^{+0.12}\right) \cdot 10^{-3} \mathrm{eV}^{2}[15]\right)$. Knowledge on the absolute mass scale comes from e.g. beta decay, $0 \nu \beta \beta$ or cosmology.

One can estimate possible values of $m_{\beta \beta}$ as a function of the lightest neutrino mass given the experimental knowledge about $\Delta m_{i j}^{2}$ and the measured rotation angles and allowing the Majorana phases to take any value. Three cases can be discriminated [19].

- If the lightest mass is larger than all $\Delta m_{i j}^{2}$, all $m_{i}$ are similar (degenerate masses).

- If $m_{1}$ is the smallest mass (normal mass hierarchy) the 3 terms of $m_{\beta \beta}$ can cancel for $m_{1} \leq \sqrt{\Delta m_{21}^{2}}$. For $m_{1} \approx 0$ typical values are a few $\mathrm{meV}$.

- If $m_{3}$ is the smallest mass (inverted hierarchy, $\Delta m_{31}^{2}<0$ ) there exists for $m_{\beta \beta}$ a lower bound of $\left(19_{-1.5}^{+1.7}\right) \mathrm{meV}$ and an upper bound of $50 \mathrm{meV}[14]$.

If additional sterile neutrinos exist, i.e. if there are additional terms in Eq. 6, then even for the inverted hierarchy no lower bound exists [20, 21].

In summary, $0 \nu \beta \beta$ - together with other input from e.g. neutrino oscillation and LHC experiments - provides an important window to extensions of the Standard Model with lepton number violation.

\section{Experimental sensitivity on $T_{1 / 2}^{0 \nu}$}

All (but one) searches have so far only observed event counts in the region of interest around $Q_{\beta \beta}$ which are consistent with the expectation from background $\lambda_{\mathrm{bkg}}$. This number - if it scales with the detector mass $M$ - is given by

$$
\lambda_{\mathrm{bkg}}=M \cdot t \cdot B \cdot \Delta E
$$

Here $t$ is the measurement time, $B$ is the so called background index given typically in cnts $/(\mathrm{keV} \cdot \mathrm{kg} \cdot \mathrm{yr})$, and $\Delta E$ is the width of the search window which depends on the experimental energy resolution. Note that this equation is only an approximation. Experiments normally take the (nonlinear) shape of the background spectrum in a fit into account.

The non-observation is converted to an upper limit on the number of signal events $\lambda_{\text {sig }}$ which is related to the half life $T_{1 / 2}^{0 \nu}$ of a given isotope $A$ by

$$
\lambda_{\text {sig }}=\ln 2 \cdot N_{\mathrm{Avg}} \cdot \epsilon \cdot \eta \cdot M \cdot t /\left(m_{A} \cdot T_{1 / 2}^{0 \nu}\right) .
$$

1 To be more precise: the amplitude has dimension mass ${ }^{-5}=M_{B}^{-4} \cdot M_{F}^{-1}$ with $M_{B}$ being the mass of a scalar or vector exchange particle like the $W$ boson. 
Table 1. List of phase space factor (Eq. 10p, $Q_{\beta \beta}$, natural abundance of $\beta \beta$ isotope, half life of $2 \nu \beta \beta$, and experiments for the most interesting $0 \nu \beta \beta$ isotopes. Half lives for $2 \nu \beta \beta$ are taken from [1, 2], $Q_{\beta \beta}$ for ${ }^{136} \mathrm{Xe}$ from [22], and all other numbers from [4]. The $G^{0 \nu}$ values have been scaled to the same nuclear radius and $g_{A}$ coupling.

\begin{tabular}{lccccl}
\hline isotope & $\begin{array}{c}G^{0 \nu} \\
{\left[\frac{10^{-14}}{\mathrm{yr}}\right]}\end{array}$ & $\begin{array}{c}Q_{\beta \beta} \\
{[\mathrm{keV}]}\end{array}$ & $\begin{array}{c}\text { nat. ab. } \\
{[\%]}\end{array}$ & $\begin{array}{c}T_{1 / 2}^{2 \nu} \\
{\left[10^{20} \mathrm{y}\right]}\end{array}$ & experiments \\
\hline${ }^{48} \mathrm{Ca}$ & 6.3 & 4273.7 & 0.187 & 0.44 & CANDLES \\
${ }^{76} \mathrm{Ge}$ & 0.63 & 2039.1 & 7.8 & 15 & GERDA, Majorana Demonstr. \\
${ }^{82} \mathrm{Se}$ & 2.7 & 2995.5 & 9.2 & 0.92 & SuperNEMO, Lucifer \\
${ }^{100} \mathrm{Mo}$ & 4.4 & 3035.0 & 9.6 & 0.07 & MOON, AMoRe \\
${ }^{116} \mathrm{Cd}$ & 4.6 & 2809.1 & 7.6 & 0.29 & Cobra \\
${ }^{130} \mathrm{Te}$ & 4.1 & 2530.3 & 34.5 & 9.1 & CUORE \\
${ }^{136} \mathrm{Xe}$ & 4.3 & 2457.8 & 8.9 & 21 & EXO, Next, Kamland-Zen \\
${ }^{150} \mathrm{Nd}$ & 19.2 & 3367.3 & 5.6 & 0.08 & SNO+, DCBA/MTD \\
\hline
\end{tabular}

$N_{\text {Avg }}$ is the Avogadro constant, $\epsilon$ the signal detection efficiency, $\eta$ the mass fraction of the $0 \nu \beta \beta$ isotope, and $m_{A}$ the molar mass of the isotope.

If $\lambda_{\text {bkg }}<1$ the experimental sensitivity scales with $M \cdot t$ while for $\lambda_{\text {bkg }}>>1$ the e.g. $90 \%$ C.L. limit on the half life (assuming there is no signal) is given by

$$
T_{1 / 2}^{0 \nu}(90 \% C L)>\frac{\ln 2}{1.64} \frac{N_{\mathrm{Avg}}}{m_{A}} \epsilon \cdot \eta \cdot \sqrt{\frac{M \cdot t}{B \cdot \Delta E}} .
$$

If systematic errors become important e.g. if the energy resolution or the spectral background shape is not well known, then the sensitivity is reduced.

\section{The nuclear matrix element}

$\left(T_{1 / 2}^{0 \nu}\right)^{-1}$ is the product of three factors: a phase space factor $G^{0 \nu}$, a nuclear matrix element $M^{0 \nu}$ and a particle physics factor.

If the exchange of light Majorana neutrinos is dominating, $T_{1 / 2}^{0 \nu}$ for a given isotope $A$ is [23]

$$
\left[T_{1 / 2}^{0 \nu}(A)\right]^{-1}=G^{0 \nu}\left(Q_{\beta \beta}, Z\right) \cdot\left|M^{0 \nu}(A)\right|^{2} \frac{m_{\beta \beta}^{2}}{m_{e}^{2}}
$$

Here, $m_{e}$ is the electron mass. Values for $G^{0 \nu}$ are listed in Tab. 1. Obviously, $M^{0 \nu}(A)$ is needed to compare results from different isotopes or to extract information about the particle physics parameter.

The $M^{0 \nu}$ calculations are difficult and can only be done using approximations. Traditionally, interacting shell model (ISM) [24, 25] and quasi particle random phase approximation (QRPA) calculations have been performed [27, 33]. Recently new approaches like the interacting boson model (IBM) [29, 30, 31], the generating coordinate model (GCM, also called energy density functional EDF) 28] and the projected Hartree-Fock-Bogoliubov (pHFB) method [32] have been applied.

The results of these calculations are shown in Fig. 1. The following statements can be made concerning the status:

- $M^{0 \nu}(A)$ varies slowly with $A$. This might be due to the fact that only neighboring neutrons in a nucleus contribute to the decay [24, 33]. 


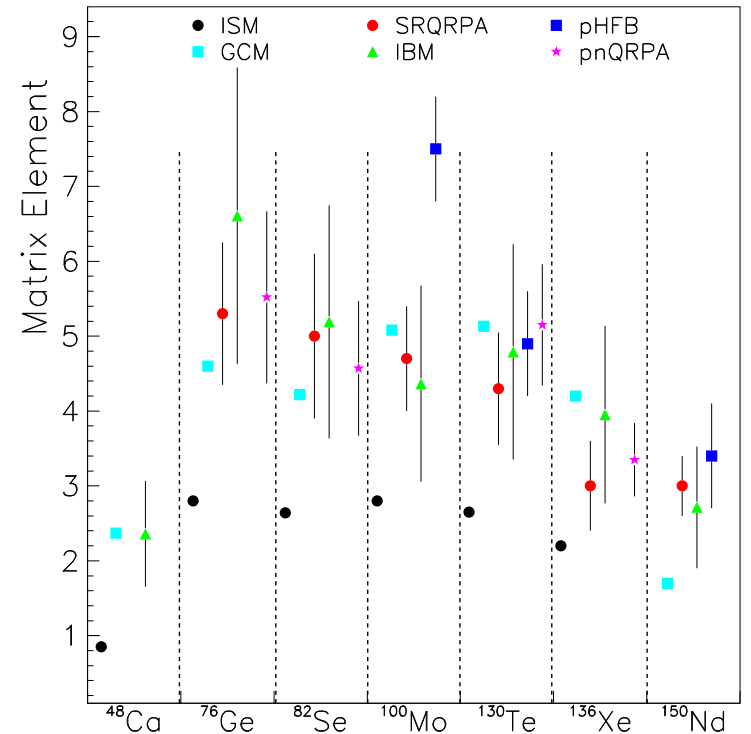

Figure 1.

Nuclear matrix element calculations for $0 \nu \beta \beta$ for light neutrino exchange. ISM $=$ Interacting shell model 24, 25, SRQRPA $=$ self-consistent renormalized quasi-particle random phase approximation [26], pnQRPA = proton-neutron quasi particle random phase approximation [27, GCM = generating coordinate method [28, IBM = interacting boson model [29, 30, 31] (matrix elements are scaled by 1.18 to estimate the effect if the UCOM short range correlation instead of the Jastrow type would have been used 9], in 30] an error of $30 \%$ is estimated), $\mathrm{pHBF}=$ projected Hartree-Fock-Bogoliubov model [32].

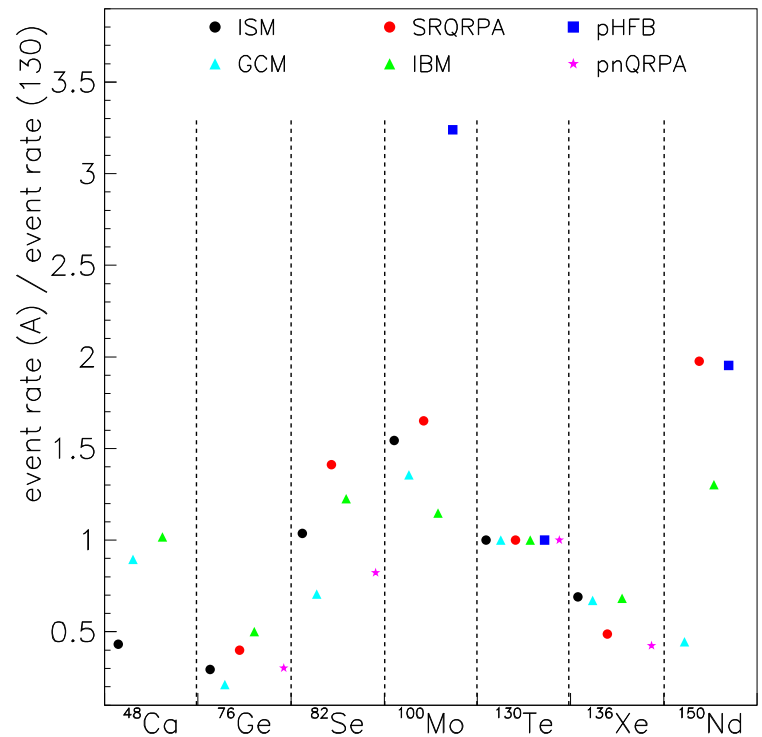

Figure 2. Ratio of expected $0 \nu \beta \beta$ events per $\mathrm{kg}$ target mass for the different matrix element models normalized to ${ }^{130} \mathrm{Te}$.

- For the ISM, all values are systematically lower than for other methods. Possible reasons for this effect are discussed in the literature [24, 34].

- For a given isotope the calculations spread by typically a factor of 2-3, i.e. a factor of 4-9 for $T_{1 / 2}^{0 \nu}$. Some groups estimate a possible range for $M^{0 \nu}$ (see indicated errors in Fig. 1). However it is unclear what to quote as confidence interval for theoretical calculations.

- The role of short range correlations has been studied and the UCOM correction has emerged as favorable [35]. Alternatively, a self consistent implementation was first applied to SRQRPA [33] and later to other methods [36, 32, and resulted in small changes.

- Experimental input can cause a sizable shift of the result. For example charge exchange reaction measurements of ${ }^{150} \mathrm{Nd}\left({ }^{3} \mathrm{He}, \mathrm{t}\right)$ and ${ }^{150} \mathrm{Sm}\left(\mathrm{t},{ }^{3} \mathrm{He}\right)$ [37 result in a quenching factor of 0.75 for the $g_{A}$ coupling and hence a reduction of the matrix element by $25 \%$ for ${ }^{150} \mathrm{Nd}$ [38. In this calculation, deformation was treated for the first time in a QRPA calculation. For ${ }^{76} \mathrm{Ge}$ and ${ }^{76} \mathrm{Se}$, the proton and neutron valence orbital occupancies have been measured [39, 40]. If the models are adjusted to reproduce these values, the ISM result increases by $15 \%$ [36] while the QRPA results are reduced by about $20 \%$ [41, 42]. Hence the difference 
between ISM and QRPA becomes half as large.

In order to see whether some isotopes are better suited for $0 \nu \beta \beta$ searches from a theoretical point of view, the number of expected decays for a given exposure (in units of $\mathrm{kg} \cdot \mathrm{yr}$ ) can be compared using Eqs. 8 and 10 without the factors $\epsilon$ and $\eta$. The $A$ dependent parameters are then the phase space factor, the matrix element and the molar mass. For the comparison it is sufficient to look at the ratio of decay rates and in this case, some of the systematic effects of the matrix element calculations cancel since there are typically correlations among the isotopes for a given method. ${ }^{2}$ Fig. 2 shows these ratios for the different models normalized to the decay rate of ${ }^{130} \mathrm{Te}$. One sees that ${ }^{76} \mathrm{Ge}$ is less favorable. The expected decays per $\mathrm{kg}$ vary between $20 \%$ and $50 \%$ of the rate of ${ }^{130} \mathrm{Te}$. In other words: if all experimental parameters (number of background events, efficiency, etc.) were the same then one would need a factor of 2-5 more target mass in a ${ }^{76} \mathrm{Ge}$ experiment to have the same sensitivity. In reality, all experimental parameters like the energy resolution and the background have to considered as well.

\section{Current experimental situation}

In the last decade mainly three experiments contributed to $0 \nu \beta \beta$ searches. Heidelberg-Moscow was for more than a decade the most sensitive one and reported evidence for this decay. The two others were Cuoricino and NEMO-3. Before, IGEX reported with an exposure of $8.8 \mathrm{~kg} \cdot \mathrm{yr}$ a limit of $T_{1 / 2}^{0 \nu}>1.57 \cdot 10^{25}$ yr for ${ }^{76} \mathrm{Ge}(90 \%$ C.L.) [43]. Currently EXO-200, Kamland-Zen, and GERDA are taking data. All experiments are discussed in this section.

\subsection{The Heidelberg-Moscow experiment}

The Heidelberg-Moscow experiment operated between 1990 and 2003 five germanium detectors made out of isotopically enriched material $\left(\simeq 86 \%{ }^{76} \mathrm{Ge}, 11 \mathrm{~kg}\right)$. The diodes were mounted in copper cryostats with copper, lead, and polyethylene shielding. The total exposure was $71.7 \mathrm{~kg} \cdot \mathrm{yr}$ and the average count rate in the interval $2-2.1 \mathrm{MeV}$ was about $0.17 \mathrm{cnts} /(\mathrm{keV} \cdot \mathrm{kg} \cdot \mathrm{yr}$ ) (for the period 1995-2003). The energy resolution (full width at half maximum, FWHM) was about $3.5 \mathrm{keV}$ at $Q_{\beta \beta}$ which is the best value of all $0 \nu \beta \beta$ experiments. Part of the collaboration finds evidence for a peak at $Q_{\beta \beta}$ with $28.75 \pm 6.86$ events which converts to $T_{1 / 2}^{0 \nu}=\left(1.19_{-0.23}^{+0.37}\right) \cdot 10^{25} \mathrm{yr}$ 6]. Note that only a statistical error is quoted. Another study finds that e.g. extending the energy window used in the data fit increases this background and hence decreases the signal count by up to $40 \%$ (Tab. 3.8 and 4.6 of reference [44]).

In a later publication [7] the claim was strengthened by a pulse shape analysis which preferentially selected $0 \nu \beta \beta$ events due to their localized energy deposition in the detectors. Backgrounds from gammas with multiple Compton scatterings exhibit different pulse shapes. The background is reduced to a surprisingly low level of $\approx 0.015 \mathrm{cnts} /(\mathrm{keV} \cdot \mathrm{kg} \cdot \mathrm{yr})$. The final fit reports a yield of $11.32 \pm 1.75$ signal events and the ratio $11.32 / 1.75=6.5$ is called the significance of the peak (Fig. 9b in [7]). The signal yield was then converted to $T_{1 / 2}^{0 \nu}=\left(2.23_{-0.31}^{+0.44}\right) \cdot 10^{25} \mathrm{yr}$. There are several problems with this analysis.

- The fit error on the signal count is too small. The smallest $68 \%$ Poisson credibility interval is between 8.1 and 15.2 for a probability distribution which peaks at 11.3, i.e. a factor of 2 larger than the quoted interval. Due to the existing (small) background the $\pm 1 \sigma$ interval should become even larger.

- The probability that the background ( $\simeq 2.2$ events in the central $3 \mathrm{keV}$ of the peak) fluctuates to the observed number of 13 events or more is $5 \cdot 10^{-7}$ which converts to a

2 This can be seen for example from Tab. 1 of reference [26. For every isotope, 20 different calculations are listed which vary by typically a factor of 2 for a given $A$. If 20 ratios $M^{0 \nu}(A) / M^{0 \nu}\left(A^{\prime}\right)$ for two isotopes $A$ and $A^{\prime}$ are calculated, the variation is reduced to $30 \%$. 
significance of about $5 \sigma$. Systematic effects like the uncertainty of the background might reduce this value.

- In the conversion to $T_{1 / 2}^{0 \nu}$ using Eq. (8), an efficiency $\epsilon$ of $100 \%$ is used although no value is explicitly quoted. All but three events in the peak are part of an earlier selection (labelled "HNR+NN" in [7]). For the latter the efficiency was $62 \%$ [45]. Hence one expects also for this analysis a value much smaller than $100 \%$.

The central $T_{1 / 2}^{0 \nu}$ value and the errors are consequently not correct in [7] and the significance is smaller than quoted although still high.

\subsection{The Cuoricino experiment}

Cuoricino [46] operated $62 \mathrm{TeO}_{2}$ crystals with a total mass of $40.7 \mathrm{~kg}\left(11.3 \mathrm{~kg}\right.$ of $\left.{ }^{130} \mathrm{Te}\right)$ between 2003 and 2008. At a temperature of $\leq 10 \mathrm{mK}$, the heat capacitance is very low and an energy deposition inside a crystal results in an increase of typically $0.1 \mathrm{mK} / \mathrm{MeV}$ which is measured with Neutron Transmutation Doped germanium thermistors. The latter have a resistance of $100 \mathrm{M} \Omega$ and show a strong temperature dependence which converts to a dependence of $3 \mathrm{M} \Omega / \mathrm{MeV}$. This bolometric technique has been proven to work with a good resolution FWHM of typically $6-10 \mathrm{keV}$ at $2.6 \mathrm{MeV}$.

The total ${ }^{130} \mathrm{Te}$ exposure was $19.75 \mathrm{~kg} \cdot \mathrm{yr}$. The background at $Q_{\beta \beta}$ was $0.17 \mathrm{cnts} /(\mathrm{keV} \cdot \mathrm{kg} \cdot \mathrm{yr})$ if normalized to the total mass, i.e. similar to Heidelberg-Moscow. No signal was found and a lower limit of $T_{1 / 2}^{0 \nu}>2.8 \cdot 10^{24} \mathrm{yr}$ (at 90\% C.L.) was set for ${ }^{130} \mathrm{Te}$. This limit is not sensitive enough to scrutinize the Heidelberg-Moscow result.

\subsection{The NEMO-3 experiment}

In NEMO-3 [4] thin foils made out of 7 different $\beta \beta$ isotopes (9 $\mathrm{kg}$ in total) were located in a drift chamber with a magnetic field. Outside of the drift region was a calorimeter made out of plastic scintillator blocks with photo multiplier tube (PMT) readout (FWHM for electrons $15 \% / \sqrt{E[\mathrm{MeV}]})$. The $0 \nu \beta \beta$ reconstruction efficiency is only about $8 \%$. On the other hand, the topological event reconstruction largely reduces backgrounds from locations other than the source foil as well as internal decays with gammas or alphas. Only $2 \nu \beta \beta$ events with poor energy reconstruction can not be discriminated. The background is about $1.2 \cdot 10^{-3} \mathrm{cnts} /(\mathrm{keV} \cdot \mathrm{kg} \cdot \mathrm{yr})$ at $3 \mathrm{MeV}\left(\approx Q_{\beta \beta}\right.$ of ${ }^{100} \mathrm{Mo}$ and $\left.{ }^{82} \mathrm{Se}\right)$. For all isotopes $2 \nu \beta \beta$ half lives are reported with impressive signal to background ratios of up to 76 . For $0 \nu \beta \beta$ decay, the $90 \%$ C.L. limits are $T_{1 / 2}^{0 \nu}>1.0 \cdot 10^{24} \mathrm{yr}$ for ${ }^{100} \mathrm{Mo}$ and $T_{1 / 2}^{0 \nu}>3.2 \cdot 10^{23}$ yr for ${ }^{82} \mathrm{Se}$. Again, NEMO-3 is not sensitive enough to confirm or reject the Heidelberg-Moscow claim.

\subsection{The Kamland-Zen experiment}

In Kamland-Zen [3] a balloon of $1.54 \mathrm{~m}$ radius made out of $25 \mu \mathrm{m}$ thick nylon is inserted into the Kamland detector and filled with xenon doped scintillator $\left(\simeq 290 \mathrm{~kg}{ }^{136} \mathrm{Xe}\right)$. The energy (FWHM $\simeq 10 \%$ at $Q_{\beta \beta}$ ) and position of the decay (resolution $\sigma \simeq 15 \mathrm{~cm} / \sqrt{E(\mathrm{MeV})}$ ) is reconstructed with PMTs located at a radius of $9 \mathrm{~m}$ which cover $34 \%$ of the solid angle.

Since the scintillator is very pure and can be doped easily with several $\%$ xenon, this experiment has the largest target mass and lowest background - if normalized to the total mass ${ }^{3}$ However the energy resolution is the poorest.

Kamland-Zen started data taking in 2011. An unexpected background peak at about $2.6 \mathrm{MeV}$ is dominating the spectrum around $Q_{\beta \beta}=2.458 \mathrm{MeV}$. It limits the experimental sensitivity and the best explanation for the origin is ${ }^{110 m} \mathrm{Ag}\left(T_{1 / 2}=250 \mathrm{~d}\right)$ cosmogenically produced in ${ }^{136} \mathrm{Xe}$ while it was above ground. This background is expected to be reduced to a negligible level

3 The Xe mass fraction is $\approx 2.5 \%$ and the fiducial volume is $\approx 43 \%$. 
by an ongoing scintillator purification campaign. After an exposure of 78 days first results are $T_{1 / 2}^{0 \nu}>5.7 \cdot 10^{24}$ yr (at $90 \%$ C.L.) and $T_{1 / 2}^{2 \nu}=(2.38 \pm 0.14) \cdot 10^{21} \mathrm{yr}$ [3]. Like for Cuoricino and NEMO-3 this limit is not yet sensitive enough to scrutinize Heidelberg-Moscow.

Additional $700 \mathrm{~kg}$ of Xe with $90 \%$ enrichment are available by the end of 2012 and are expected to be deployed in a cleaner balloon in the near future. Kamland-Zen is therefore expected to be the first experiment with ton scale isotope mass. In the more distant future it is foreseen to improve the energy reconstruction with light collectors attached to the PMTs and a new liquid scintillator [48].

\subsection{The EXO-200 experiment}

EXO-200 [2] operates a liquid xenon TPC of $40 \mathrm{~cm}$ diameter and $40 \mathrm{~cm}$ length $(175 \mathrm{~kg}$ of liquid $\mathrm{Xe}, 100 \mathrm{~kg}$ fiducial mass). A wire plane in the middle is biased to $-8 \mathrm{kV}$ such that electrons drift to one of the two ends where the $(x, y)$ position of the electron cloud is reconstructed with 2 planes of wires and the total charge is measured. The produced scintillation light is detected with large area avalanche photo diodes behind the charge collecting wires.

Since there is a strong anti-correlation between the light amplitude and the ionization signal, combining both into a new quantity greatly improves the energy resolution to $\mathrm{FWHM} \approx 3.9 \%$ at $Q_{\beta \beta}$. The background is at a quite low level of $\simeq 1.5 \cdot 10^{-3} \mathrm{cnts} /(\mathrm{keV} \cdot \mathrm{kg} \cdot \mathrm{yr})$ after a geometrical cut on the electron cloud is applied to discriminate Compton scattered photon events.

EXO has recently published results on the $2 \nu \beta \beta$ half life $T_{1 / 2}^{2 \nu}=(2.1 \pm 0.2) \cdot 10^{21} \mathrm{yr}$ and a limit on the $0 \nu \beta \beta$ half life $T_{1 / 2}^{0 \nu}>1.6 \cdot 10^{25} \mathrm{yr}$ (at $90 \%$ C.L.) of ${ }^{136} \mathrm{Xe}$ [2].

This result can be used to test the claim of Heidelberg-Moscow. For a given matrix element calculation the expected number of signal events for EXO can be estimated from Eq. 8 and 10 . The experimental numbers of EXO $(32.5 \mathrm{~kg} \cdot \mathrm{yr}$ exposure, $55 \%$ reconstruction efficiency $40.6 \%$ enrichment) and Heidelberg-Moscow (28.75 \pm 6.86 events, $71.7 \mathrm{~kg} \cdot \mathrm{yr}$ exposure, efficiency $100 \%$, enrichment $86 \%)$ enter. EXO-200 reports $n_{\mathrm{obs}}=1\left(n_{\mathrm{obs}}=5\right)$ events in an energy window of $\pm 1 \sigma( \pm 2 \sigma)$.

A Bayesian formulation can be applied with a null hypothesis $(H$ : EXO observes only background) and an alternative hypothesis $(\bar{H}$ : EXO observes a $0 \nu \beta \beta$ signal with scaled Heidelberg-Moscow event counts). The priors for the expected number of signal events $\pi_{s}$ and for background events $\pi_{b}$ are Gaussian and the priors for both hypothesis are set to $\pi(H)=\pi(\bar{H})=0.5$. Mean and sigma of $\pi_{s}$ are listed in Tab. 2. Mean and sigma of the background are $4.1 \pm 0.3(7.5 \pm 0.7)$ events in the $\pm 1 \sigma( \pm 2 \sigma)$ energy window.

The posterior probability is then

$$
p(\bar{H})=\frac{\pi(\bar{H}) \cdot p(D \mid \bar{H})}{\pi(\bar{H}) \cdot p(D \mid \bar{H})+\pi(H) \dot{p}(D \mid H)}
$$

with

$$
\begin{aligned}
p(D \mid \bar{H}) & =\int \pi_{s}(x) \pi_{b}(y) P\left(n_{\text {obs }} \mid x+y\right) \mathrm{d} x \mathrm{~d} y \\
p(D \mid H) & =\int \pi_{b}\left(y \mid \lambda_{b}, \sigma_{b}\right) P\left(n_{\text {obs }} \mid y\right) \mathrm{d} y
\end{aligned}
$$

Here $P\left(n_{\text {obs }} \mid y\right)$ is the Poisson function for mean $y$ and $n_{\text {obs }}$ observed events. The results for $p(\bar{H})$ are listed in Tab. 2. For the Tübingen-Bratislava calculations (labelled "QRPA max" and "QRPA min") the maximum and minimum of 20 ratios $M^{0 \nu}\left({ }^{136} \mathrm{Xe}\right) / M^{0 \nu}\left({ }^{76} \mathrm{Ge}\right)$ are calculated

\footnotetext{
4 An efficiency is not explicitly given in [2] but an effective value can be estimated using Eq. 8 and the information from the publication.
} 
Table 2. Bayesian posterior probabilities $p(\bar{H})$ using EXO-200 data for the hypothesis that the $0 \nu \beta \beta$ signal of Heidelberg-Moscow is correct. Probabilities are given for different matrix element calculations and for the $\pm 1 \sigma$ and $\pm 2 \sigma$ energy windows.

\begin{tabular}{lcccc}
\hline method & \multicolumn{2}{c}{ in $\pm 1 \sigma$ window } & \multicolumn{2}{c}{ in $\pm 2 \sigma$ window } \\
& expected signal evts. & $p(\bar{H})$ in $\%$ & expected signal evts. & $p(\bar{H})$ in $\%$ \\
\hline QRPA max & $4.4 \pm 1.1$ & 4 & $6.1 \pm 1.5$ & 6 \\
QRPA min & $2.8 \pm 0.7$ & 11 & $3.9 \pm 0.9$ & 16 \\
ISM & $10.6 \pm 2.5$ & 0.1 & $14.8 \pm 3.5$ & 0.2 \\
GCM & $14.3 \pm 3.4$ & 0.03 & $19.9 \pm 4.8$ & 0.05 \\
pnQRPA & $6.3 \pm 1.5$ & 1 & $8.8 \pm 2.1$ & 2 \\
IBM & $6.1 \pm 1.5$ & 1 & $8.6 \pm 2.1$ & 2 \\
\hline
\end{tabular}

from the values of Tab. 1 of reference [26]. This procedure was suggested by one of the authors [49]. For the other models the ratios of the central values shown in Fig. 1 are taken. None of the results strengthen the hypothesis of a $0 \nu \beta \beta$ signal. The ISM and GCM calculations strongly disfavor them while the exclusion for the Tübingen-Bratislava calculations are not very strong. The reported EXO-200 data were taken within a period of 7 months. Hence more stringent statements are expected soon.

EXO-200 is approved to run for 4 more years. For a following phase the spectroscopic identification of the daughter nucleus of Xe is foreseen. In this case the experiment will be background free. Only $2 \nu \beta \beta$ events with poorly reconstructed energy can obscure a signal.

\subsection{The GERDA experiment}

GERDA uses the germanium detectors of Heidelberg-Moscow and IGEX and - in a second phase - new ones. The $\beta \beta$ emitter mass is about $13 \mathrm{~kg}$ in the first phase. The detectors are supported by a minimal amount of material with low radioactivity in a $4 \mathrm{~m}$ diameter cryostat filled with liquid argon. Argon serves as cooling medium and shield against external radioactivity. The latter is complemented by $3 \mathrm{~m}$ of water which is instrumented with PMTs to veto background from muons by the detection of their Cherenkov light.

GERDA started commissioning in 2010 and found an unexpected large background from ${ }^{42} \mathrm{Ar}$ which could be reduced by avoiding electrical fields around the detectors and by an encapsulation of the diodes. Since November 2011 the first phase of data taking is ongoing. The background is at the level of $0.02 \mathrm{cnts} /(\mathrm{keV} \cdot \mathrm{kg} \cdot \mathrm{yr})$ and hence almost an order of magnitude smaller than the equivalent number of Heidelberg-Moscow. Due to a data blinding procedure no result on $0 \nu \beta \beta$ is expected before spring 2013. A preliminary result for $2 \nu \beta \beta$ of $T_{1 / 2}^{2 \nu}=(1.88 \pm 0.10) \cdot 10^{21} \mathrm{yr}$ is reported [50].

A second phase will start early 2013 with additional new detectors $(\simeq 18 \mathrm{~kg}$ of $\left.{ }^{76} \mathrm{Ge}\right)$. The background is expected to be reduced from currently $0.02 \mathrm{cnts} /(\mathrm{keV} \cdot \mathrm{kg} \cdot \mathrm{yr})$ to $0.001 \mathrm{cnts} /(\mathrm{keV} \cdot \mathrm{kg} \cdot \mathrm{yr})$ due to a liquid argon instrumentation and a different detector type with enhanced pulse shape discrimination power.

\section{Other future experiments}

The past and running experiments have been discussed above but there are others under construction or as R\&D efforts. They use additional isotopes, and various other detection mechanisms and background reduction methods, see Tab. 3 .

One important experimental parameter is the fraction $\eta$ of the $0 \nu \beta \beta$ isotope (see Eq. (9) and Tab. 11. ${ }^{130} \mathrm{Te}$ is the only one with a large natural abundance. For all other elements enrichment 
of the $0 \nu \beta \beta$ isotope is mandatory. ${ }^{136} \mathrm{Xe}$ is easiest to enrich with gas centrifuges since this is the heaviest isotope and it is already a gas. Other materials like ${ }^{76} \mathrm{Ge}$ can be converted to a gas $\left(\mathrm{GeF}_{4}\right)$ and then processed. For calcium and neodymium this path is currently not available and $\mathrm{R} \& \mathrm{D}$ on alternative methods is ongoing.

Another important number is $Q_{\beta \beta}$. Larger values are not only better because of larger $G^{0 \nu}$ but also because the background from natural decay chains falls off fast beyond $2.6 \mathrm{MeV}$. Experiments using ${ }^{76} \mathrm{Ge}$ have to compensate by careful material selection and good energy resolution.

The experiments can be grouped into 2 classes. In calorimetric experiments only the total energy (ionization or scintillation) is measured. In tracking experiments the two electrons are measured independently, i.e. the angular distribution between the electrons is also known. The latter is interesting to study the origin of the underlying physics in case $0 \nu \beta \beta$ is observed.

In the following, the experiments under construction are discussed.

CUORE is a continuation of Cuoricino with close to $1000 \mathrm{TeO}_{2}$ crystals of $750 \mathrm{~g}$ each (in total $\simeq 200 \mathrm{~kg}$ of ${ }^{130} \mathrm{Te}$ ). The crystal production is almost finished and all major hardware items are ready or close to. A first tower with 52 crystals (CUORE-0) has been assembled and data taking in the Cuoricino cryostat was scheduled for July 2012 [51].

After a commissioning phase CUORE is expected to start in 2015.

Majorana will operate similar germanium diodes like GERDA $\left(\simeq 27 \mathrm{~kg}\right.$ of $\left.{ }^{76} \mathrm{Ge}\right)$ in vacuum in a compact cryostat made out of electro-formed copper. This self-made copper is expected to have a factor $>100$ smaller thorium, uranium and radium contaminations compared to commercial copper such that the background index is about $0.001 \mathrm{cnts} /(\mathrm{keV} \cdot \mathrm{kg} \cdot \mathrm{yr})$. The shielding is completed by commercial copper, lead, and polyethylene. Operations should start in 2013.

In a later phase a combined GERDA and Majorana germanium experiment with order ton scale mass is envisioned.

CANDLES operates 96 scintillating $\mathrm{CaF}_{2}$ crystals $\left(0.3 \mathrm{~kg}\right.$ of $\left.{ }^{48} \mathrm{Ca}\right)$ in a liquid scintillator. Both are in an acrylic container inside a water tank with PMTs. Data taking started in 2011 but the sensitivity is limited due to the small target mass. Successful R\&D on ${ }^{48} \mathrm{Ca}$ enrichment is crucial for this approach.

NEXT is a high pressure xenon gas TPC (1.1 m diameter, $1.4 \mathrm{~m}$ length, pressure 10-15 bar, mass $90-130 \mathrm{~kg}{ }^{136} \mathrm{Xe}$ ). The time of the $0 \nu \beta \beta$ decay is determined by the detection of the (primary) scintillation light with PMTs. The deposited energy and event topology is reconstructed from the ionization signal. The drifting electron cloud passes at the end a volume of higher electric field such that the electrons are moderately accelerated. Consequently, they can excite xenon but not ionize it. The resulting (secondary) scintillation light (electroluminescence) is proportional to the number of electrons and detected with the same PMTs (for energy reconstruction) and with a plane of SiPMs (for position reconstruction). The energy resolution FWHM is expected to be $<1 \%$ at $Q_{\beta \beta}$ and the event topology will allow to reject backgrounds very effectively to a level of $8 \cdot 10^{-4} \mathrm{cnts} /(\mathrm{keV} \cdot \mathrm{kg} \cdot \mathrm{yr})$.

Construction will start in 2013 and physics data taking in 2015.

SuperNEMO is a planned continuation of NEMO-3 with much improved performance (factor 4 reconstruction efficiency, factor 2 in energy resolution, factor 6 in background). A demonstrator module with $7 \mathrm{~kg}$ of ${ }^{82} \mathrm{Se}$ is under construction and expected to start data taking in 2014.

SNO+ dissolves $\approx 1$ ton ${ }^{\text {nat }} \mathrm{Nd}$ in 780 tons of liquid scintillator $\left(44 \mathrm{~kg}\right.$ of ${ }^{150} \mathrm{Nd}$ ). The scintillator is in a $12 \mathrm{~m}$ diameter acrylic vessel which is surrounded by pure water in an $18 \mathrm{~m}$ diameter water tank. The latter holds the 95008 inch PMTs for light detection. An energy resolution FWHM of $\approx 7 \%$ at $Q_{\beta \beta}$ is expected with an extremely low background of $\leq 10^{-6} \mathrm{cnts} /(\mathrm{keV} \cdot \mathrm{kg} \cdot \mathrm{yr})$ if normalized to the total scintillator mass. The main background 
Table 3. Selection of $0 \nu \beta \beta$ experiments.

\begin{tabular}{|c|c|c|c|c|c|}
\hline experiment & isotope & mass $[\mathrm{kg}]$ & method & start / end & ref. \\
\hline \multicolumn{6}{|c|}{ past experiments } \\
\hline Heidelberg-Moscow & ${ }^{76} \mathrm{Ge}$ & 11 & ionization & -2003 & 6] \\
\hline Cuoricino & ${ }^{130} \mathrm{Te}$ & 11 & bolometer & -2008 & 46 \\
\hline NEMO-3 & ${ }^{100} \mathrm{Mo},{ }^{82} \mathrm{Se}$ & 7,1 & track. +calorim. & -2011 & [47] \\
\hline \multicolumn{6}{|c|}{ current experiments } \\
\hline EXO-200 & ${ }^{136} \mathrm{Xe}$ & 175 & liquid TPC & 2011- & 2 \\
\hline Kamland-Zen & ${ }^{136} \mathrm{Xe}$ & 330 & liquid scintil. & 2011- & 3 \\
\hline GERDA-I/ GERDA-II & ${ }^{76} \mathrm{Ge}$ & $15 / 35$ & ionization & 2011-/ 2013- & 50 \\
\hline CANDLES & ${ }^{48} \mathrm{Ca}$ & 0.35 & scint. crystal & 2011- & 52 \\
\hline \multicolumn{6}{|c|}{ funded experiments } \\
\hline NEXT & ${ }^{136} \mathrm{Xe}$ & 100 & gas TPC & 2015 & 53 \\
\hline Cuore0/ Cuore & ${ }^{130} \mathrm{Te}$ & $10 / 200$ & bolometer & $2012-/ 2015-$ & 54 \\
\hline Majorana Demo. & ${ }^{76} \mathrm{Ge}$ & 30 & ionization & 2013 & 55 \\
\hline SuperNEMO demo./total & ${ }^{82} \mathrm{Se}$ & $7 / 100$ & track.+calorim. & $2014-/ ? ?$ & 56 \\
\hline $\mathrm{SNO}+$ & ${ }^{150} \mathrm{Nd}$ & 44 & liquid scint. & 2013 & [57] \\
\hline \multicolumn{6}{|c|}{ proposal, proto-typing } \\
\hline Cobra & ${ }^{116} \mathrm{Cd}$ & & solid TPC & & 58 \\
\hline Lucifer & ${ }^{82} \mathrm{Se}$ & & bolom. +scint. & & 59 \\
\hline DCBA/MTD & ${ }^{150} \mathrm{Nd}$ & 32 & tracking & & 60 \\
\hline MOON & ${ }^{82} \mathrm{Se},{ }^{100} \mathrm{Mo}$ & $30-480$ & track. +scint. & & 61 \\
\hline AMoRE & ${ }^{100} \mathrm{Mo}$ & 100 & bolom. +scint. & & 62 \\
\hline Cd exp. & ${ }^{116} \mathrm{Cd}$ & & scint. & & 63 \\
\hline
\end{tabular}

is $2 \nu \beta \beta$ due to the poor energy resolution and the relatively short half life $T_{1 / 2}^{2 \nu}$ of ${ }^{150} \mathrm{Nd}$ (see Tab. 1). Scintillator filling and doping with $\mathrm{Nd}$ is expected for 2013 . Since ${ }^{150} \mathrm{Nd}$ can not be enriched effectively at the moment, $\eta$ is small which limits the $\mathrm{SNO}+$ sensitivity.

Lucifer is an R\&D effort which investigates scintillating crystals with $\beta \beta$ emitters like ZnSe which are operated as bolometers. The simultaneous detection of phonons and photons allows to identify backgrounds from e.g. surface events which are expected to dominate in CUORE.

Other ongoing R\&D efforts are not discussed here. References are listed in Tab. 3 .

\section{Comparison of experiments}

For a comparison of the sensitivities of the experiments a relative scaling factor for the different matrix elements and phase spaces has to be applied. This factor can be estimated from Fig. 2. The values used here are $f_{A}(\mathrm{Ge})=0.35(0.2-0.5), f_{A}(\mathrm{Se})=1.1(0.7-1.4)$, $f_{A}(\mathrm{Mo})=2.1(1.1-3.2), f_{A}(\mathrm{Te})=1, f_{A}(\mathrm{Xe})=0.55(0.4-0.7)$ and $f_{A}(\mathrm{Nd})=1.2(0.4-2.0)$. The numbers in parentheses are the full range.

If the number of background events is large, Eq. (9) can be used to estimate the experimental sensitivity. A relative figure-of-merit can then be defined as

$$
\mathrm{FOM}=f_{A} \cdot \epsilon \cdot \eta \cdot \sqrt{\frac{M}{B \cdot \Delta E}}
$$

The relative $T_{1 / 2}^{0 \nu}$ sensitivity scales with the live time $t$ of an experiment like FOM. $\sqrt{t}$. Tab. 4 lists the performance numbers of the experiments discussed above. "Kamland-Zen2" is the improved experiment after the purification of the scintillator (assumed factor 5 smaller total background) 
Table 4. Comparison of relative figure-of-merit (FOM), lower half life limit $T_{1 / 2}^{0 \nu}$ after $4 \mathrm{yr}$ live time, and resulting upper limit on $m_{\beta \beta}$. For $m_{\beta \beta}$, the entire range of matrix element values including the indicated error bars in Fig. 1 are used. $f_{A}$ is the average scale factor for a given isotope taken from Fig. 2. $\Delta E$ is the energy window which is taken to be 1(2) FWHM for experiments with $>0.5 \%(<0.5 \%)$ resolution. Note that the efficiency is reduced by 0.7 if $\Delta E=1$.FHWM. FOM is defined in the text. Masses are total masses or fiducial masses. The background and enrichment fraction has to be scaled accordingly.

\begin{tabular}{|c|c|c|c|c|c|c|c|c|c|}
\hline exp. & $\begin{array}{c}\text { mass } \\
{[\mathrm{kg}]}\end{array}$ & $f_{A}$ & $\begin{array}{c}\text { bkg. } \\
{\left[\frac{10^{-3} \mathrm{cnt}}{\mathrm{keV} \cdot \mathrm{kg} \cdot \mathrm{yr}}\right]}\end{array}$ & $\begin{array}{c}\Delta E \\
{[\mathrm{keV}]}\end{array}$ & eff. & enrich. & FOM & $\begin{array}{c}T_{1 / 2}^{0 \nu} \\
10^{25} \mathrm{yr}\end{array}$ & $\begin{array}{l}m_{\beta \beta} \\
\mathrm{meV}\end{array}$ \\
\hline \multicolumn{10}{|c|}{ past experiments } \\
\hline Hd-Moscow & 11 & 0.35 & 120 & 7 & 1 & 0.86 & 1 & 1.9 & $170-530$ \\
\hline Cuoricino & 41 & 1 & 170 & 16 & 0.9 & 0.28 & 1 & 0.4 & $210-500$ \\
\hline \multicolumn{9}{|c|}{ running experiments } & $310-900$ \\
\hline EXO-200 & 100 & 0.55 & 1.5 & 100 & 0.55 & 0.81 & 6 & 4.2 & $75-170$ \\
\hline Kaml.-Zen & 12800 & 0.55 & 0.05 & 250 & 0.31 & 0.023 & 4 & 2.6 & $90-220$ \\
\hline Kaml.-Zen2 & 12800 & 0.55 & 0.01 & 250 & 0.31 & 0.06 & 22 & 15 & $40-90$ \\
\hline GERDA-I & 15 & 0.35 & 20 & 8 & 0.8 & 0.86 & 2 & 3.9 & $120-370$ \\
\hline GERDA-II & 35 & 0.35 & 1 & 6 & 0.85 & 0.88 & 20 & 18 & $60-170$ \\
\hline \multicolumn{10}{|c|}{ experiments under construction } \\
\hline Major.-Dem. & 30 & 0.35 & 1 & 6 & 0.9 & 0.9 & 20 & 17 & $60-170$ \\
\hline CUORE & 750 & 1 & 10 & 12 & 0.9 & 0.27 & 19 & 7.5 & $50-110$ \\
\hline $\mathrm{SNO}+$ & 780000 & 1.5 & 0.0002 & 230 & 0.33 & $5.6 \mathrm{E}-5$ & 3 & 0.8 & $100-240$ \\
\hline NEXT & 100 & 0.55 & 0.8 & 25 & 0.25 & 0.9 & 9 & 5.2 & $70-160$ \\
\hline \multicolumn{10}{|c|}{ proposed experiments } \\
\hline S.NEMO & 100 & 1.1 & 0.1 & 200 & 0.2 & 0.9 & 14 & 6.9 & $55-140$ \\
\hline Lucifer & 100 & 1.1 & 1 & 10 & 0.9 & 0.5 & 50 & 19 & $33-85$ \\
\hline
\end{tabular}

and the upgrade to one ton xenon mass. For comparison, the FOM numbers, the expected $90 \%$ C.L. $T_{1 / 2}^{0 \nu}$ limits for $4 \mathrm{yr}$ of live time, and the corresponding $m_{\beta \beta}$ limits are given. For the latter, the entire spread of the matrix elements of Fig. 1 including the error bars are used.

For running (and past) experiments the achieved performance values are used which might improve with time. For the others the anticipated performance numbers are taken.

As a graphical representation, the relative sensitivity of the experiments as a function of live time is shown in Fig. 3 This value is calculated from Eq. (8) by

$$
\hat{T}_{1 / 2}^{0 \nu}>\frac{f_{A} \cdot \epsilon \cdot \eta \cdot M \cdot t}{\Psi(B \cdot \Delta E \cdot M \cdot t)}
$$

Here $\Psi\left(\lambda_{\mathrm{bkg}}\right)$ is the "average" $90 \%$ C.L. upper limit of the number of signal events for $\lambda_{\mathrm{bkg}}$ background events calculated according to the method discussed in 64.

A few comments should be made concerning the interpretation of Tab. 4 and Fig. 3.

- The factor $f_{A}$ and hence FOM has a full spread (not $\sigma$ ) of $\approx \pm 30 \%-70 \%$. Thus the curves in Fig. 3 could be replaced by bands which would make the figure however unreadable.

- The sensitivities discussed here are calculated for $0 \nu \beta \beta$ exclusion limits. For a positive signal claim, the situation is different. A good energy resolution like the ones for germanium or bolometer experiments will allow to identify a narrow line at the correct energy. This is extremely valuable if the existence of the rarest ever observed decay will be claimed. 


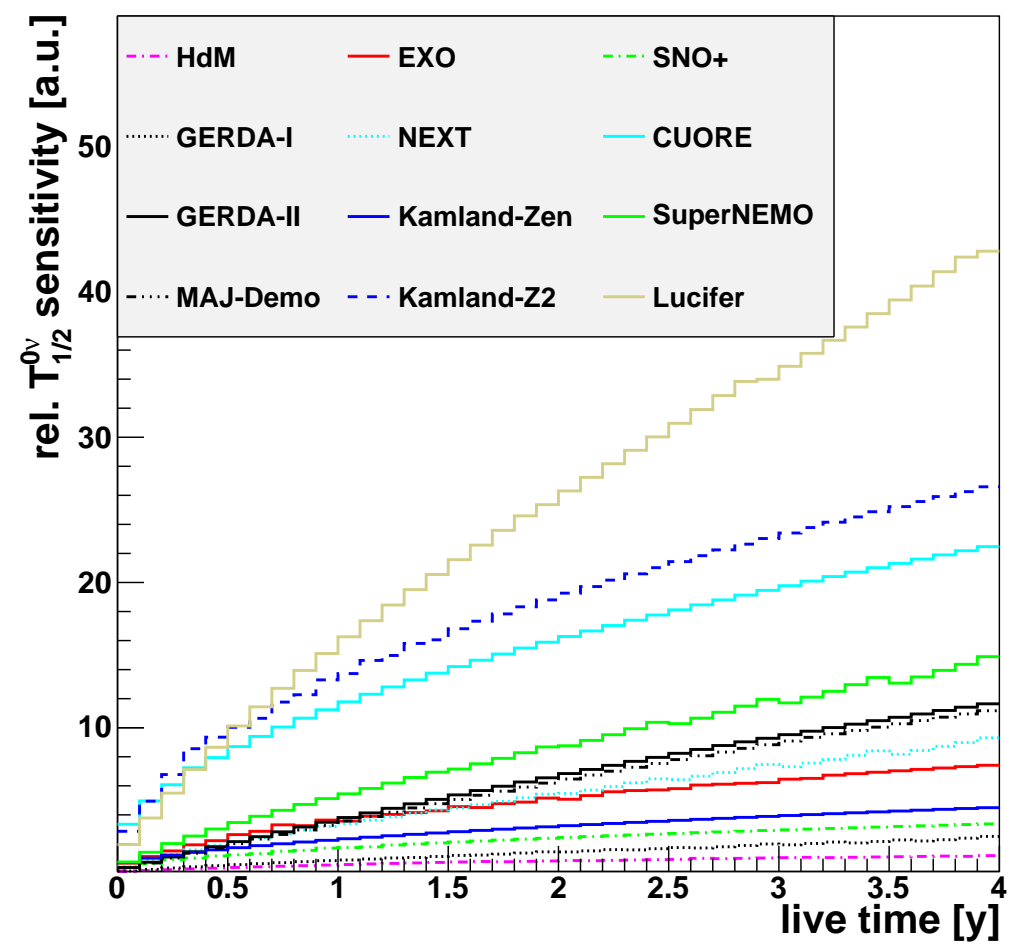

Figure 3. Relative experimental sensitivity for the $0 \nu \beta \beta$ half life limit versus live time for different experiments.

- To estimate relative sensitivities for $m_{\beta \beta}$, the inverse square root has to be taken of the curves shown in Fig. 3 and the FOM numbers in Tab 4 . The variation due to the spread of the matrix elements is reduced in this case as can be seen from the last column of Tab. 4. i.e. the spread for the lower or upper value of the $m_{\beta \beta}$ interval is reduced.

If instead limits for other particle physics parameters are calculated, the scaling will be different. For heavy neutrino exchange, the mass limit scales with $\sqrt{\hat{T}_{1 / 2}^{0 \nu}}$. The factor $f_{A}$ will change in this case but for most models the variations is less than $30 \%$ [65, 66].

- The $2 \nu \beta \beta$ background is irreducible and can only be avoided with an energy resolution $\sigma<1-2 \%$ at $Q_{\beta \beta}$. This requirement depends of course strongly on $T_{1 / 2}^{2 \nu}$ which varies by a factor of 300 for the isotopes considered.

- Of the ongoing experiments, Kamland-Zen(2) should have the largest potential. However a low background bolometer experiment like Lucifer or an improved CUORE experiment will be even more sensitive. The sensitivity of them would still grow almost linearly after 4 yr (see Fig. 3).

- Germanium experiments can be competitive to e.g. current xenon experiments with a factor of 3 more mass despite the fact that the phase space factor is small. Required is however a factor of 10 lower background than the one of GERDA-I.

- Systematic effects like the uncertainty on the fiducial volume or the knowledge of the background level are not taken into account here.

The goal of many searches is to reach a sensitivity equivalent to $m_{\beta \beta} \approx 19 \mathrm{meV}$ which would 
cover practically the entire expected range for the inverted neutrino mass hierarchy. For ${ }^{76} \mathrm{Ge}$, this corresponds to half lives of $(1.5-15) \cdot 10^{27}$ years if the entire span of matrix elements of Fig. 1 is taken into account. These values should be compared to the expected sensitivity of GERDA-II or Majorana Demonstrator of about $1.7 \cdot 10^{26} \mathrm{y}$. This demonstrates that exploring the entire mass band of the inverted hierarchy is a long term enterprise $5^{5}$ The equivalent numbers for ${ }^{136} \mathrm{Xe},{ }^{82} \mathrm{Se}$, and ${ }^{130} \mathrm{Te}$ are $(0.6-3.5) \cdot 10^{27} \mathrm{yr},(0.6-3.8) \cdot 10^{27} \mathrm{yr}$, and $(0.5-2.5) \cdot 10^{27} \mathrm{yr}$, respectively. They seem to be easier to reach if one compares them to the $T_{1 / 2}^{0 \nu}$ limits in Tab. 4 .

Considering the example of the Heidelberg-Moscow claim and the uncertainties of the matrix elements, one can conclude that a signal has to be observed in several isotopes to establish $0 \nu \beta \beta$.

To improve the credibility of the result a blind analysis should be performed. This technique is nowadays standard in particle physics experiments and should be adopted in this field as well.

It is also worth mentioning that continuously new ideas for $0 \nu \beta \beta$ experiments are coming up 67] and the one who will make a discovery might not be listed in this publication.

\section{Summary}

Neutrinoless double beta decay violates lepton number and the experimental programs are therefore on equal footing to proton decay searches. It might also be the only practical process which allows to test whether neutrinos are Majorana particles. The motivation for several large efforts in this field is therefore obvious.

For a long time, the Heidelberg-Moscow experiment has dominated the field and its claim of a $0 \nu \beta \beta$ signal has not been scrutinized since 2001. The recent EXO-200 limit does not support this claim but can not refute it due to the spread of the nuclear matrix element calculations. GERDA does not suffer from such uncertainties and will unblind the data in spring 2013. Then the combined data from GERDA, EXO-200 and Kamland-Zen should be sensitive enough for a meaningful test.

Beyond this next step, experiments want to reach a sensitivity to explore the $m_{\beta \beta}$ region of the inverted neutrino mass hierarchy. This will eventually require ton scale experiments. Kamland-Zen will be the first one but whether the entire range will be covered will depend on the achievable background level.

For a convincing claim of a $0 \nu \beta \beta$ signal a good energy resolution is important and the detection with several isotopes.

\section{Acknowledgements}

The motivation for this article and many improvements are due to discussions with several colleagues. I want to thank especially Allen Caldwell, Karl Tasso Köpfle, and Stefan Schönert.

\section{References}

[1] Barabash A S 2010 Phys. Rev. C81 035501 (Preprint arXiv:1003.1005)

[2] Auger A et al. 2012 Phys. Rev. Lett. 109032505 (Preprint arXiv:1205.5608)

[3] Gando A et al. 2012 Phys. Rev. C 85045504 (Preprint arXiv:1201.4664)

[4] Rodejohann W 2011 Int. J. Mod. Phys. E20 1833 (Preprint arXiv:1106.1334)

[5] Vergados J D, Ejiri H and Simkovic F 2012 Rept. Prog. Phys. 75106301 (Preprint arXiv:1205.0649)

[6] Klapdor-Kleingrothaus H V et al. 2004 Phys. Lett. B 586198

[7] Klapdor-Kleingrothaus H V and Krivosheina I V 2006 Mod. Phys. Lett. A 211547

[8] Avignone F T, Elliott S R and Engel J 2008 Rev. Mod. Phys. 80481 (Preprint arXiv:0708.1033)

[9] Gomez-Cadenas J J et al. 2012 Riv. Nuovo Cim. 3529 (Preprint arXiv:1109.5515)

[10] Suhonen J 2012 J. Phys. Conf. Ser. 375042026

[11] Rukhadze N et al. 2012 J. Phys. Conf. Ser. 375042020

[12] Belli P et al. 2012 J. Phys. Conf. Ser. 375042024

5 To reach a limit of $10^{28} \mathrm{yr}$, a 1 ton ${ }^{76} \mathrm{Ge}$ experiment has to operate for $10 \mathrm{yr}$ with a background which is a factor of 10 smaller than the one of GERDA-II. 
[13] Fang D L et al. 2012 Phys. Rev. C 85035503 (Preprint arXiv:1111:6862)

[14] Bilenky S M and Giunti C 2012 Mod. Phys. Lett. A27 1230015 (Preprint arXiv:1203.5250)

[15] Beringer J et al. (Particle Data Group) 2012 Phys. Rev. D86 010001

[16] Schechter J and Valle J 1982 Phys. Rev. D25 2951

[17] M Duerr M L and Merle A 2011 JHEP 1106091 (Preprint arXiv:1105.0901)

[18] Aaij R et al. (LHCb collaboration) 2012 Phys. Rev. D85 112004 (Preprint arXiv:1201.2238)

[19] Feruglio F, Strumia A and Vissani F 2003 Phys. Lett. B 659359 (Preprint arXiv:hep-ph/0201291)

[20] Barry J, Rodejohann W and Zhang H 2011 JHEP 110791 (Preprint arXiv:1105.3911)

[21] Giunti C et al. arXiv:1210.5715

[22] Redshaw M et al. 2007 Phys. Rev. Lett. 98053003

[23] Doi M, Kotani T and Takasugi E 1985 Prog. Theor. Phys. Suppl. 831

[24] Menendez J et al. 2009 Nucl. Phys. A818 139 (Preprint arXiv:0801.3760)

[25] Menendez J et al. 2011 J. Phys. Conf. Ser. 312072005

[26] Faessler A, Rodin V and Simkovic F ArXiv:1206.0464

[27] Suhonen J and Civitarese O 2010 Nucl. Phys A847 207

[28] Rodriguez T R and Martinez-Pinedo G 2010 Phys. Rev. Lett. 105252503 (Preprint arXiv:1008.5260)

[29] Barea J and Iachello F 2009 Phys. Rev. C79 044301

[30] Barea J and Iachello F 2011 Nucl. Phys. B (Proc. Suppl.) 2175

[31] F Iachello J B and Kotila J 2011 AIP Conf. Proc. 141762

[32] Rath P K et al. 2010 Phys. Rev. C82 064310 (Preprint arXiv:1104.3965)

[33] Simkovic F et al. 2009 Phys. Rev. C79 055501 (Preprint arXiv:0902.0331)

[34] Escuderos A et al. 2010 J. Phys. G37 125108 (Preprint arXiv:1001.3519)

[35] Civitarese O and Suhonen J 2009 J. Phys. Conf. Ser. 173012012

[36] Menendez J et al. 2009 Phys. Rev. C80 048501 (Preprint arXiv:0905.1705)

[37] Guess C J et al. 2011 Phys. Rev. C83 064318 (Preprint arXiv:1105.0677)

[38] Fang D L et al. 2011 Phys. Rev. C83 034320 (Preprint arXiv:1101.2149)

[39] Schiffer J P et al. 2008 Phys. Rev. Lett. 100112501 (Preprint arXiv:0710.0719)

[40] Kay B P et al. 2009 Phys. Rev. C79 021301 (Preprint arXiv:0810.4108)

[41] Simkovic F et al. 2009 Phys. Rev. C79 015502 (Preprint arXiv:0812.0348)

[42] Suhonen J and Civitarese O 2008 Phys. Lett. B668 277

[43] Aalseth C E et al. 2002 Phys. Rev. D 65092007 (Preprint arXiv:hep-ex/0202026)

[44] Chkvorets O PhD thesis University of Heidelberg, arXiv:0812.1206

[45] Klapdor-Kleingrothaus H V et al. 2004 Nucl. Instr. Meth. A 522371

[46] Andreotti E et al. 2011 Astropart.Phys. 34822 (Preprint arXiv:1012.3266)

[47] Simard L 2012 J. Phys. Conf. Ser. 375042011

[48] Inoue K Results from Kamland-Zen, talk given at Neutrino 2012 conference

[49] Rodin V Private communication

[50] Grabmayr P Results from GERDA, talk given at Neutrino 2012 conference

[51] Pedretti M Status of the CUORE Experiment, talk given at Neutrino 2012 conference

[52] Ogawa I et al. 2012 J. Phys. Conf. Ser. 375042018

[53] Alvarez V et al. 2012 JINST 7 T06001 (Preprint arXiv:1202.0721)

[54] Gorla P 2012 J. Phys. Conf. Ser. 375042013

[55] Wilkerson J F et al. 2012 J. Phys. Conf. Ser. 375042010

[56] Barabash A 2012 J. Phys. Conf. Ser. 375042012

[57] Hartnell J 2012 J. Phys. Conf. Ser. 375042015

[58] Oldorf C 2012 J. Phys. Conf. Ser. 375042022

[59] Cardani L 2012 J. Phys. Conf. Ser. 375042016

[60] Ishihara N 2012 J. Phys. Conf. Ser. 375042017

[61] Fushimi K et al. 2010 J. Phys. Conf. Ser. 203012064

[62] Bhang H et al. 2012 J. Phys. Conf. Ser. 375042023

[63] Barabash A S 2011 JINST 6 P08011 (Preprint arXiv:1108.2771)

64] Gomez-Cadenas J J et al. 2011 JCAP 20117 (Preprint arXiv:1010.5112)

[65] Faessler A et al. 2011 Phys. Rev. D83 113015 (Preprint arXiv:1103.2504)

[66] Deppisch F and Päs H 2007 Phys. Rev. Lett. 9823250 (Preprint arXiv:hep-ph/0612165)

[67] Gomez-Cadenas J J et al. 2012 J. Cosm. Astropart. Phys. 2012037 (Preprint arXiv:1110.6133) 